\title{
DIFERENCIAS ENTRE JUGADORES DE FÚTBOL, BALONCESTO Y TENIS DE MESA EN LA CAPACIDAD DE SALTO VERTICAL Y HORIZONTAL
}

DIFFERENCES AMONG SOCCER, BASKETBALL AND TABLE TENNIS PLAYERS IN VERTICAL AND HORIZONTAL JUMP CAPACITY

\section{Picabea, J.M. ${ }^{1}$, Yanci, J. ${ }^{1}$}

1. Facultad de Ciencias de la Actividad Física y del Deporte, Universidad del País Vasco, UPV/EHU, Vitoria-Gasteiz, España.

Correo electrónico: javier.yanci@ehu.es

Código UNESCO: 2402.05

Clasificación Consejo de Europa: 3. Biomecánica del deporte, 6. Fisiología del ejercicio.

Recibido el 30 de marzo de 2014

Aceptado el 28 de junio de 2015

Correspondencia:

Dr. Javier Yanci Irigoyen.

Departamento de Educación Física y Deporte, Facultad de Ciencias de la Actividad Física y del Deporte, Universidad del País Vasco, UPV/EHU.

Lasarte, 71, 01007. Vitoria, España

Telf. +0034945013529

\section{RESUMEN}

Los objetivos de este estudio fueron analizar las diferencias existentes en la capacidad de salto vertical y horizontal en función del deporte practicado (futbol, baloncesto y tenis de mesa) y determinar si existía algún tipo de relación entre las capacidades de salto horizontal y vertical. 37 deportistas de 16-18 años (16 jugadores de fútbol, 11 de baloncesto y 10 de tenis de mesa) fueron evaluados en la capacidad de salto vertical y horizontal, tanto bilateral como unilateral. No se encontraron diferencias significativas en la capacidad de 
salto vertical, pero si en la capacidad de salto horizontal, siendo los jugadores de baloncesto los que más saltaban. Se encontró una correlación significativa entre el salto horizontal y vertical a dos piernas $(r=0,724, p<0,01)$. A pesar de los resultados obtenidos, son necesarios más estudios donde se analicen las diferencias en la capacidad de salto horizontal entre distintos deportes con el fin de corroborar los resultados obtenidos.

Palabras clave: correlación, potencia, salto bilateral, salto unilateral, fuerza.

\section{ABSTRACT}

The aims of this study were to analyze the differences in the vertical and horizontal jump ability depending on the sport practiced (soccer, basket and table tennis) and to determinate the correlation between the horizontal and vertical jumps. 37 young athletes (16-18 years, 16 soccer players, 11 basketball players and 10 table tennis player) of were evaluated on the vertical and horizontal jump ability, both bilaterally and unilaterally. No significant differences in vertical jumping ability were found, but in the horizontal jump, significant differences were found, being the basketball players obtained the best performance. Correlation between the two legs horizontal and vertical jump was found $(r=0.724, p<0.01)$. However, more studies are needed comparing horizontal jump capacity between sports, to corroborate the obtained results.

KEYWORDS: correlation, power, bilateral jump, unilateral jump, strength.

\section{INTRODUCCIÓN}

La fuerza muscular y la potencia de las extremidades inferiores de los deportistas tienen una gran importancia en el rendimiento en varios deportes como pueden ser el baloncesto $(1,2)$, el voleibol $(3,4)$, el tenis de mesa $(5)$, el balonmano $(6,7)$, el fútbol $(8,9,10)$, el ciclismo (11) y el ski $(12)$, entre otros. Esta cualidad se manifiesta en multitud de acciones específicas como pueden ser la capacidad de realizar acciones rápidas, sprints, saltos y cambios de dirección (13). Por lo tanto, para realizar estas acciones de forma eficaz se necesita un buen nivel de fuerza muscular y potencia. De la misma forma, se ha definido que un adecuado nivel de fuerza muscular puede reducir la probabilidad de sufrir lesiones (14). Si bien la capacidad de generar fuerza en 
la musculatura del tren inferior tiene gran importancia en la mayor parte de deportes, varias investigaciones se han centrado en su valoración en tres modalidades concretas como son el futbol (15), el baloncesto (16) y el tenis de mesa (5). En el caso del fútbol, tal y como indica Bueno (17), durante un partido un jugador puede llegar a realizar 15 saltos verticales máximos dentro del área, pudiendo ser alguno de ellos decisivo para el resultado del partido. En el baloncesto, tal y como indica López (18), las acciones que habitualmente se realizan son explosivas y muchas de ellas a una intensidad máxima, permitiendo así la ejecución de gestos técnicos inalcanzables para otros jugadores (rebotes, mates...). En el tenis de mesa, los movimientos del tren inferior son esenciales para lograr una correcta posición, siendo estos movimientos cortos y explosivos debido a los rápidos cambios de dirección que se dan en el partido (5).

Para la medición de la fuerza explosiva del tren inferior, los test que se han utilizado con frecuencia han sido los test de salto vertical, siendo su utilización muy frecuente en modalidades deportivas como en fútbol (8, 14, 19), el baloncesto $(1,20)$ o el tenis de mesa $(5,21)$. La capacidad de salto vertical se ha analizado también en varios rangos de edad y distintos niveles competitivos dentro de un mismo deporte $(8,22,23)$. Nikolaidis et al. (8) analizaron la capacidad de salto con contra movimiento (CMJ) en el fútbol con jugadores de 8 a 31 años de edad. Huertas et al. (22) compararon el salto sin contra movimiento (SJ) y $\mathrm{CMJ}$ en el fútbol diferenciando las categorías benjamín, alevín, infantil, juvenil y absoluto. Hellín et al. (23) analizaron la capacidad de salto en el baloncesto con jugadores profesionales y amateurs. Sin embargo, muchas de las acciones que se dan en la práctica deportiva como los sprints, los cambios de dirección y los saltos, requieren de una producción de fuerza no solo el eje vertical, sino también en el eje horizontal $(24,25)$. Así mismo, dependiendo de la modalidad deportiva, se requiere de una propulsión unilateral para realizar acciones específicas (25). En la literatura científica son pocos los estudios en los que se analice la capacidad de generar fuerza en el eje horizontal $(7,15)$, a pesar de que se ha observado que los test de salto horizontal son validos y fiables (25). 
Por otro lado, las comparaciones entre distintas modalidades deportivas en la capacidad de salto vertical han tenido una importante repercusión en la literatura científica $(11,26,27,28)$. Cowley et al. (27) compararon la capacidad de salto vertical entre jugadoras femeninas de baloncesto y fútbol, de la misma forma que Gomes et al. (29), aunque estos últimos lo hicieron con jugadores masculinos. Marques et al. (28) compararon la capacidad de salto vertical entre jugadores de fútbol, balonmano y fútbol sala. Rouis et al. (30) compararon la capacidad de salto vertical entre jugadores de voleibol y estudiantes de educación física. Por otro lado, Yanci y Los Arcos (11), compararon la capacidad de salto vertical entre atletas y ciclistas de élite. La mayor parte de los estudios donde se compara la fuerza muscular de las extremidades inferiores en distintas modalidades han utilizados test de salto en el eje vertical. Sin embargo, no hemos encontrado ningún estudio que analice las diferencias en función del deporte practicado mediante la capacidad de salto horizontal.

Por lo tanto, los objetivos de este estudio fueron por un lado, observar si existían diferencias en la capacidad de salto vertical y horizontal dependiendo de la disciplina deportiva practicada (futbol, baloncesto y tenis de mesa) en deportistas de 16-18 años, y por otro, determinar si existía una relación entre el rendimiento en el salto vertical (SV) y el salto horizontal (SH).

\section{MÉTODO}

\section{Participantes}

En este estudio participaron 37 deportistas masculinos de categoría juvenil $\left(16,9 \pm 0,9\right.$ años, 70,4 $\left.\pm 7,2 \mathrm{~kg}, 1,8 \pm 0,1 \mathrm{~m}, 22,9 \pm 2,1 \mathrm{~kg} \cdot \mathrm{m}^{-2}\right)$, de los cuales dieciséis eran jugadores de fútbol (FUT) de categoría juvenil de honor, once eran jugadores de baloncesto (BAL) de categoría junior masculino de segundo año y diez jugadores de tenis de mesa (TME) de la primera división vasca (Tabla 1). 
Tabla 1. Características generales de los participantes

\begin{tabular}{lccc}
\hline & $\begin{array}{l}\text { FUT }(\mathrm{n}=16) \\
\text { Media } \pm \mathrm{DT}\end{array}$ & $\begin{array}{c}\text { BAL }(\mathrm{n}=11) \\
\text { Media } \pm \mathrm{DT}\end{array}$ & $\begin{array}{c}\text { TME }(\mathrm{n}=10) \\
\text { Media } \pm \mathrm{DT}\end{array}$ \\
\hline Edad (años) & $17,1 \pm 0,1$ & $16,1 \pm 0,6$ & $17,2 \pm 0,9$ \\
Altura $(\mathrm{m})$ & $1,8 \pm 0,1$ & $1,8 \pm 0,1$ & $1,7 \pm 0,1$ \\
Masa $(\mathrm{Kg})$ & $72,0 \pm 6,6$ & $69,6 \pm 10,8$ & $69,6 \pm 5,5$ \\
IMC $\left(\mathrm{Kg} \cdot \mathrm{m}^{-2}\right)$ & $23,1 \pm 2,2$ & $21,9 \pm 2,6$ & $23,6 \pm 1,8$ \\
\hline DT = Desviación típica, & IMC = Índice de Masa Corporal, FUT = grupo de futbolistas, BAL = \\
grupo de jugadores de baloncesto, TME = grupo de jugadores de tenis de mesa.
\end{tabular}

Los criterios de inclusión fueron tener una edad comprendida entre los 16 y los 18 años, tener licencia federativa en vigor expedida por las Federaciones Españolas de Fútbol, de Baloncesto o de Tenis de Mesa y no encontrarse lesionado en el momento de la investigación. Todos los participantes fueron informados de los objetivos y procedimientos de la investigación y, aceptaron voluntariamente formar parte de ella previa firma del consentimiento informado, la cual firmaron los padres, madres o tutores legales en el caso de los jugadores menores de edad. Los participantes realizaban una media de 2-3 sesiones de entrenamiento semanales y disputaban un partido oficial cada semana. El estudio se realizó bajo el consentimiento del club al que pertenecían. Todos los procedimientos siguieron las pautas marcadas por la Declaración de Helsinki (2013) y se respetó lo establecido en la Ley Orgánica de Protección de Datos de Carácter Personal (LOPDCP).

\section{Procedimiento}

Los test se realizaron a mitad de temporada de los tres equipos, en un día de entrenamiento. Los participantes realizaron en el mismo día las mediciones antropométricas básicas de peso y altura y los test salto vertical con contra movimiento con manos libres a dos piernas (VCMJAS) y a una pierna, dominante (VCMJASD) y no dominante (VCMJASND). Del mismo modo se realizaron test de salto horizontal con contra movimiento con manos libres a dos piernas (HCMJAS) y una pierna, dominante (HCMJASD) y no dominante 
(HCMJASND). En la sesión de toma de datos de cada equipo, el calentamiento previo realizado fue el mismo y consistió en 5 min de carrera continua y varios saltos verticales y horizontales. Todos los participantes disponían del material e indumentaria adecuada para la práctica de los test. Se consideró la pierna dominante la que cada jugador, tanto de fútbol, baloncesto y tenis de mesa, utilizaba de forma natural para el golpeo de un balón de fútbol (31).

\section{Batería de Test}

Test de salto en la vertical: Los participantes realizaron 3 VCMJAS a dos piernas y 3 saltos con contra movimiento con cada una de las piernas, VCMJASD y VCMJASND (32). El descanso entre saltos fue de 45 segundos. Todos los saltos se realizaron con manos libres durante toda la fase del salto. Durante la fase de impulso, la flexión de rodillas debía llegar alrededor de los $90^{\circ}$, permitiéndose una ligera flexión de tronco. Las piernas debían estar extendidas durante el primer contacto con el suelo en la fase de aterrizaje para posteriormente flexionarlas y así amortiguar el impacto. Se realizaron 3 registros de cada tipo de salto, obteniendo 9 registros por cada participante. Para medir la altura de vuelo se utilizó una plataforma de contacto (Optojump ${ }^{\circledR}$, Microgate Engineering, Bolzano, Italia).

Test de salto en la horizontal: Los participantes realizaron 3 saltos horizontales a dos piernas (HCMJAS) y 3 saltos horizontales con cada una de las piernas, HCMJASD y HCMJASND. Todos los saltos se realizaron con las manos libres durante toda la fase del salto. El descanso entre saltos fue de 45 segundos. Para la realización del salto, los participantes se colocaban detrás de una línea y realizando una flexión de rodillas, realizaban el salto en la horizontal, marcando como distancia de salto la distancia entre la línea de inicio desde la que se realizaba el salto y el apoyo del talón más próximo a la línea (25). Se realizaron 3 registros de cada tipo de salto, obteniendo 9 registros por cada participante. Para medir la distancia del salto se utilizó una cinta métrica colocada en el suelo. 


\section{Análisis estadístico de los datos}

Los resultados se presentan como media \pm desviación típica (DT) de la media. Todas las variables mostraron una distribución normal según el test de Kolmogorov-Smirnov. Para el análisis de los resultados en los distintos test se consideró únicamente el mejor de los tres registros. Se calculó el coeficiente de variación (CV) de todas las variables analizadas para determinar la estabilidad de la medición entre los intentos $[C V=(D T / m e d i a) \times 100]$. Se realizó un análisis de varianza (ANOVA) de un factor, junto con el correspondiente análisis pos hoc de Bonferroni para comparar los resultados obtenidos por los grupos (FUT, BAL y TME) en los diferentes protocolos de salto y una prueba $\mathrm{t}$ de muestras relacionadas para el análisis de diferencias dentro de un mismo grupo entre los saltos realizados con la pierna dominante y no dominante, tanto en los saltos en la vertical como en los saltos en la horizontal. Para calcular el déficit bilateral (LA) entre la pierna dominante (D) y no dominante (ND) en cada uno de los saltos se utilizo la fórmula: $L A=[(N D-D) / D) \times 100]$, tal y como había sido establecido anteriormente por Newton et al. (32). El tamaño del efecto (d) se calculó atendiendo al método propuesto por Cohen (33). Tamaños del efecto menores a 0,2, entre 0,2-0,5, entre 0,5-0,8 o mayores de 0,8 fueron considerados trivial, bajo, moderado o alto, respectivamente. Se calculó el coeficiente de correlación de Pearson y la regresión lineal para estudiar la relación entre las diferentes variables de salto. La significatividad estadista fue de $p<0,05$. El análisis estadístico se realizó con el programa Statistical Package for Social Sciences (versión 21,0, SPSS ${ }^{\circledR}$ Inc. Chicago, IL, EE.UU).

\section{RESULTADOS}

Los resultados para el total de la muestra en los test de salto vertical bilateral (VCMJAS), y unilaterales (VCMJASD y VCMJASND) fueron de 0,42 \pm 0,06 cm, $0,26 \pm 0,04 \mathrm{~cm}, 0,26 \pm 0,04 \mathrm{~cm}$ respectivamente y el déficit bilateral vertical (VLA) fue de $3,30 \pm 14,42 \%$. Los resultados en función del deporte practicado por los participantes se muestran en la tabla 2. No se encontraron diferencias 
significativas en la capacidad de SV en función del deporte, aunque para el VCMJAS entre los grupos de FUT y BAL y para el VCMJASD entre los grupos de BAL y TME, el tamaño del efecto fue alto $(d>0,8)$. A pesar de que no se encontraron diferencias significativas en el VLA entre los distintos grupos, el grupo BAL obtuvo un mayor VLA que el grupo TME $(d=1,06)$.

Tabla 2. Resultados en los test de salto vertical (SV) en función del deporte practicado.

\begin{tabular}{lcccccc}
\hline Variable & $\begin{array}{c}\text { FUT } \\
\text { Media } \pm \text { DT }\end{array}$ & $\begin{array}{c}\text { BAL } \\
\text { Media } \pm \text { DT }\end{array}$ & $\begin{array}{c}\text { TME } \\
\text { Media } \pm \text { DT }\end{array}$ & $\begin{array}{c}\text { Cohen d } \\
\text { FUT-BAL }\end{array}$ & $\begin{array}{c}\text { Cohen d } \\
\text { FUT-TME }\end{array}$ & $\begin{array}{c}\text { Cohen d } \\
\text { BAL-TME }\end{array}$ \\
\hline Saltos bilaterales & & & & & & 0,50 \\
VCMJAS (cm) & $0,40 \pm 0,05$ & $0,45 \pm 0,06$ & $0,42 \pm 0,06$ & 1,00 & 0,40 & 0,62 \\
VCMJAS CV (\%) & $3,09 \pm 3,20$ & $3,72 \pm 2,33$ & $5,17 \pm 3,29$ & 0,51 & 0,96 & \\
Saltos unilaterales & & & & & 0,20 & 1,00 \\
VCMJASD (cm) & $0,26 \pm 0,05$ & $0,25 \pm 0,02$ & $0,27 \pm 0,05$ & 0,2 & 0,78 & 0,69 \\
VCMJASD CV (\%) & $7,34 \pm 4,55$ & $6,46 \pm 3,85$ & $3,80 \pm 2,48$ & 0,19 & 0,00 & 0,33 \\
VCMJASND (cm) & $0,26 \pm 0,04$ & $0,27 \pm 0,03$ & $0,26 \pm 0,05$ & 0,25 & 0,00 \\
VCMJASND CV (\%) & $5,01 \pm 3,86$ & $7,04 \pm 3,57$ & $5,35 \pm 2,44$ & 0,53 & 0,09 & 0,47 \\
VLA (\%) & $3,77 \pm 16,55$ & $8,13 \pm 9,87$ & $-2,40 \pm 14,21$ & 0,26 & 0,37 & 1,06
\end{tabular}

VCMJAS = salto vertical con contra movimiento con manos libres, VCMJAS CV = Coeficiente de variación en salto vertical con contra movimiento con manos libres, VCMJASD = Salto vertical con contra movimiento con manos libres y pierna dominante, VCMJASD CV= Coeficiente de variación en salto vertical con contra movimiento con manos libres y pierna dominante, VCMJASND = Salto vertical con contra movimiento con manos libres y pierna no dominante, VCMJASND CV= Coeficiente de variación en salto vertical con contra movimiento con manos libres y pierna no dominante, VLA $=$ Déficit bilateral en el eje vertical, DT $=$ Desviación típica, FUT = grupo de fútbol, BAL = grupo de baloncesto, $T M E$ = grupo de tenis de mesa.

Los resultados de todos los deportistas en las pruebas de HCMJAS, HCMJASD y HCMJASND fueron de 2,19 $\pm 0,17 \mathrm{~m}, 1,85 \pm 0,18 \mathrm{~m}, 1,90 \pm 0,17 \mathrm{~m}$, respectivamente y el déficit bilateral horizontal (HLA) fue de 2,86 $\pm 6,05 \%$. Los resultados en función del deporte se muestran en la tabla 3. Se encontraron diferencias significativas en el HCMJAS $(p<0,01, d=1,31)$, en el HCMJASD ( $p$ $<0,01, d=1,13)$ y en el HCMJASND $(p<0,01, d=1,53)$ entre los grupos de FUT y BAL, siendo los jugadores de baloncesto los que más saltaron en ambos test. De la misma forma, se obtuvieron diferencias significativas en el HCMJASND entre el grupo BAL y $\operatorname{TME}(p<0,01, d=3,44)$, siendo los 
jugadores de baloncesto los que mayores valores consiguieron. En cuanto al HLA, no se observaron diferencias significativas entre las distintas modalidades deportivas.

Tabla 3. Resultados en los test de salto horizontal (SH) en función del deporte practicado.

\begin{tabular}{|c|c|c|c|c|c|c|}
\hline Variable & $\begin{array}{c}\text { FUT } \\
\text { Media } \pm \text { DT }\end{array}$ & $\begin{array}{c}\text { BAL } \\
\text { Media } \pm \text { DT }\end{array}$ & $\begin{array}{c}\text { TME } \\
\text { Media } \pm \text { DT }\end{array}$ & $\begin{array}{l}\text { Cohen d } \\
\text { FUT-BAL }\end{array}$ & $\begin{array}{l}\text { Cohen d } \\
\text { FUT-TME }\end{array}$ & $\begin{array}{l}\text { Cohen d } \\
\text { BAL-TME }\end{array}$ \\
\hline \multicolumn{7}{|l|}{ Saltos bilaterales } \\
\hline HCMJAS (cm) & $2,11 \pm 0,16$ & $2,32 \pm 0,12$ & $2,19 \pm 0,13$ & $1,31^{* *}$ & 0,50 & 1,08 \\
\hline HCMJAS CV (\%) & $3,79 \pm 1,72$ & $2,44 \pm 2,02$ & $0,95 \pm 0,58$ & 0,78 & 1,65 & 0,74 \\
\hline \multicolumn{7}{|l|}{ Saltos unilaterales } \\
\hline $\operatorname{HCMJASD}(\mathrm{m})$ & $1,83 \pm 0,16$ & $2,01 \pm 0,10$ & $1,71 \pm 0,11$ & $1,13^{\star *}$ & 0,75 & 3,00 \\
\hline HCMJASD CV (\%) & $3,61 \pm 2,26$ & $2,72 \pm 2,15$ & $1,86 \pm 1,85$ & 0,39 & 0,77 & 0,40 \\
\hline HCMJASND (m) & $1,85 \pm 0,15$ & $2,08 \pm 0,09$ & $1,77 \pm 0,11$ & $1,53^{* *}$ & 0,53 & $3,44^{* *}$ \\
\hline HCMJASND CV (\%) & $3,69 \pm 1,73$ & $3,27 \pm 2,05$ & $2,23 \pm 2,27$ & 0,24 & 0,84 & 0,51 \\
\hline HLA (\%) & $1,43 \pm 5,24$ & $3,89 \pm 5,90$ & $4,00 \pm 7,47$ & 0,47 & 0,49 & 0,02 \\
\hline
\end{tabular}

HCMJAS $=$ salto horizontal con contra movimiento con manos libres, HCMJAS CV $=$ Coeficiente de variación en salto horizontal con contra movimiento con manos libres, HCMJASD = Salto horizontal con contra movimiento con manos libres y pierna dominante, HCMJASD CV = Coeficiente de variación en salto horizontal con contra movimiento con manos libres y pierna dominante, HCMJASND = Salto horizontal con contra movimiento con manos libres y pierna no dominante, HCMJASND CV= Coeficiente de variación en salto horizontal con contra movimiento con manos libres y pierna no dominante, HLA = Déficit bilateral en el eje horizontal, DT = Desviación típica, FUT = grupo de fútbol, BAL = grupo de baloncesto, $\mathrm{TME}=$ grupo de tenis de mesa.

Diferencias significativas $\left({ }^{* *} p<0,01\right)$ entre grupos.

En cuanto a la correlación entre los diferentes tipos de saltos, en todas las variables se observó una correlación significativa $(p<0,01)$, excepto entre el VCMJASD y el HCMJASD por un lado y entre el VCMJASD y el HCMJASND por otro, tal y como se muestra en la tabla 4. Sin embargo, esta relación tan solo fue alta entre el VCMJAS y el HCMJAS, siendo la regresión lineal y = $1,297+2,128 x+0,116$ 
Tabla 4. Resultados de las correlaciones entre saltos en el eje vertical (SV) y horizontal (SH)

\begin{tabular}{llll}
\hline Variable & HCMJAS & HCMJASD & HCMJASND
\end{tabular}

VCMJAS

VCMJASD

VCMJASND

$0,495^{\star *}$

$0,445^{\star \star}$

horizontal con contra movimiento con manos libres y pierna dominante, HCMJASND = Salto horizontal con contra movimiento con manos libres y pierna no dominante, VCMJAS = salto vertical con contra movimiento con manos libres, $\mathrm{VCMJASD}=$ Salto vertical con contra movimiento con manos libres y pierna dominante, $\mathrm{VCMJASND}=$ Salto vertical con contra movimiento con manos libres y pierna no dominante. Correlación significativa $\left({ }^{* *} p<0,01\right)$ entre las distintas variables.

\section{DISCUSIÓN}

Los objetivos de este estudio fueron, por un lado, analizar las diferencias existentes en la capacidad de salto vertical (SV) y horizontal (SH) en función del deporte practicado (fútbol, baloncesto y tenis de mesa), en deportistas de 16-18 años y por otro lado, determinar si existía algún tipo de relación entre las capacidades de SV y SH. A pesar de que existen estudios en la literatura científica donde se analiza la capacidad de SV comparando diferentes modalidades deportivas $(11,27,28)$, no hemos encontrado estudios en los que se comparen las diferencias en la capacidad de SH en función de la modalidad deportiva practicada. En nuestro estudio, no se encontraron diferencias significativas en el SV en función del deporte. Sin embargo, sí se encontraron diferencias significativas en el $\mathrm{SH}$, siendo los jugadores de baloncesto los que más saltaban tanto con las dos piernas (HCMJAS), como con la pierna dominante (HCMJASD) y la pierna no dominante (HCMJASND). 
Atendiendo a los resultados obtenidos en nuestro estudio, no se encontraron diferencias significativas en la capacidad de SV en función del deporte ni en el salto bilateral (VCMJAS) ni unilateral (VCMJASD y VCMJASND). Estos resultados concuerdan con otros trabajos en los que compararon la capacidad de SV en función de diferentes deportes. Gomes et al. (29) compararon la capacidad de SV entre jugadores de fútbol y baloncesto y no encontraron diferencias significativas. Otro estudio realizado por Miranda da Rocha et al. (34), en el que se comparó la capacidad de SV entre jugadores de baloncesto y voleibol, tanto de categoría sénior como junior, tampoco encontraron diferencias. A pesar de que en el juego del baloncesto se producen más acciones de salto en el eje vertical (rebotes, mates, entradas a canasta, lanzamientos) (35) que en el fútbol o en el tenis de mesa, los jugadores de baloncesto de nuestro estudio no obtuvieron mejores resultados que los jugadores de las otras dos modalidades deportivas. Tricoli et al. (35) observaron una correlación significativa entre la potencia muscular y la capacidad de SV, concluyendo que a una mayor potencia muscular del tren inferior, los deportistas obtenían una mayor altura en el SV. El hecho de que la potencia muscular del tren inferior en el eje vertical es una de las cualidades que asiduamente se entrena en futbol $(9,10)$, en baloncesto $(1,2)$ y en tenis de mesa (5) ha podido provocar la ausencia de diferencias en la capacidad de SV en función de las distintas modalidades. Posiblemente, todos los deportistas realizaban ejercicios específicos de fuerza en el eje vertical, lo que provoco similares resultados en el rendimiento en el SV.

En cuanto a la capacidad de $\mathrm{SH}$, en nuestro estudio se obtuvieron diferencias significativas entre los diferentes grupos. Concretamente se encontraron diferencias significativas entre los futbolistas y los jugadores de baloncesto tanto en el HCMAS ( $p<0,01, d=1,31)$, como en el HCMJASD $(p<0,01, d=$ 1,13) y en el HCMJASND ( $p<0,01, d=1,53$ ), siendo los practicantes de baloncesto los que más saltaban. De la misma forma, los jugadores de baloncesto, mostraron mejores resultados que los jugadores de tenis de mesa en el HCMJASND ( $p<0,01, d=3,44$ ). Este es el primer estudio que hemos encontrado donde se analizan las diferencias en la capacidad de $\mathrm{SH}$ en función 
de la modalidad de práctica deportiva. La fuerza en el eje horizontal ha sido definida como una variable muy importante en acciones específicas de varios deportes $(5,15)$ y además tiene una importancia fundamental en la capacidad de aceleración y sprint $(36,37,38)$, variables que afectan al rendimiento en gran cantidad de deportes $(2,14,23,39)$. A pesar de la importancia de la fuerza horizontal en el rendimiento deportivo, se desconoce si en la propia actividad de algunos deportes se dan más acciones en el eje horizontal que en otros. Dado que las razones de que se hayan encontrado diferencias en la capacidad de $\mathrm{SH}$ en los jugadores de distintas modalidades deportivas no son claras, son necesarios más estudios que analicen tanto la capacidad de salto en este eje como las implicaciones de la fuerza horizontal en las acciones específicas de cada deporte.

En el presente estudio se encontraron correlaciones significativas $(p<0,01)$ en todos los tipos de salto, excepto entre el VCMJASD-HCMJASD y el VCMJASDHCMJASND. La mayor correlación obtenida fue entre el VCMJAS y HCMJAS $(r=0,724, p<0,01)$. Estos resultados coinciden con otros estudios $(25,40)$. Maulder y Cronin (25) encontraron una alta correlación entre el VCMJAS y el HCMJAS $(r=0,79)$ en jóvenes deportistas $(25,1 \pm 4,3$ años $)$ de varios deportes en los que predominaba el tren inferior. De la misma forma, Izquierdo et al. (40), encontraron una alta correlación entre el VCMJAS y el HCMJAS ( $r=0,83$, $p<0,01$ ) en 12 deportistas sanos de 20 años de edad. De forma similar, en el estudio realizado por Castro-Piñero et al. (41), en el cual participaron 94 sujetos, de entre 6 y 17 años de edad, físicamente activos que practicaban natación, fútbol o baloncesto con una frecuencia de 3-5 veces por semana, también encontraron una correlación significativa entre el VCMJAS y el HCMJAS $(r=0,843, p<0,01)$. Esta alta correlación entre el salto bilateral en el eje vertical y horizontal puede ser debida a que los dos test miden la potencia del tren inferior (42). Sin embargo, el hecho de que los test de salto unilateral son movimientos menos naturales y su ejecución es más complicada para los jóvenes deportistas (41), ha podido influir en la obtención de peores correlaciones en los saltos unilaterales. 
La principal limitación de este estudio fue la baja muestra en cada una de las modalidades deportivas. Además, el hecho de no haber encontrado estudios en el que se compare la capacidad de $\mathrm{SH}$ en función del deporte, ha sido un factor limitante ya que no se han podido comparar los resultados obtenidos. Por lo tanto, sería interesante realizar más investigaciones en las que se comparen más modalidades deportivas y con un número de participantes mayor con el fin de corroborar los resultados obtenidos en esta investigación.

\section{CONCLUSIONES}

Tal y como se ha podido comprobar en este estudio, no se han encontrado diferencias significativas en la capacidad de salto vertical en función de las diferentes modalidades deportivas, pero sí en la capacidad de salto horizontal. Así mismo, se ha obtenido una alta correlación entre el salto vertical y horizontal bilateral. Estas altas correlaciones entre los test, el hecho de que los test de salto horizontal discriminan entre jugadores de distintos deportes y que requieren menos material que los test de salto vertical, ponen de manifiesto que los test de salto en el eje horizontal pueden ser muy útiles para medir la potencia del tren inferior en deportistas juveniles. Sin embargo, se necesitan más estudios que comparen la capacidad de salto horizontal entre modalidades deportivas y que analicen la implicación de la fuerza horizontal en las acciones específicas de los distintos deportes con el fin de corroborar los resultados obtenidos en este estudio.

\section{AGRADECIMIENTOS}

Los autores quieren agradecer a todos los equipos que tomaron parte en el estudio, a los entrenadores por las facilidades ofrecidas y a los jugadores por el esfuerzo realizado en las pruebas de evaluación. 


\section{REFERENCIAS}

1. Delgado, P. Osoroio, A. Mancilla, R. Jerez, D. (2011). Análisis del desarrollo de la fuerza reactiva y saltabilidad, en basquetbolistas que realizan un programa de entrenamiento polimétrico. Revista Motricidad y Persona, 10, 33-44.

2. Bradic, A. Bradic, J. Pasalic, E. Markovic, G. (2009). Isokinetic leg strength profile of elite male basketball players. Journal of Strength and Conditioning Research, 23(4), 1332-1337.

3. Freitas, V.H. Nakamura, F.Y. Miloski, B. Samulski, D. Mauricio, G. (2014). Sensitivity of physiological and psychological markers to training load intensification in volleyball players. Journal of Sports Science and Medicine, 13, 571-579.

4. Parra, R. (2012). Fuerza explosiva y resistencia a la fuerza explosiva de miembros inferiores en mujeres voleibolistas de Santander. Acción, 8(15), 11-15.

5. Pradas, F. Castellar, C. Ochiana, N. (2013). Analysis of explosive an elastic-explosive strength of lower limbs in spanish young top-level table tennis players. Gymnasium, Scientific Journal of Education, Sports and Health, 14(1), 21-28.

6. Polat, S.Ç. Öz, E. Orhan, O. Yarim, I. Cetin, E. (2014). Comparison of sprint, repeated sprint and jumping parameters of different levels handball players. Ovidius University Annals, Series Physical Education and Sport Science, Movement and Health, 14(2), 543-547.

7. Vila, H. Fernández, J.J. Rodríguez, F.A. (2007). Evolución de la condición física en jugadoras de balonmano en las categorías infantil, cadete y juvenil. Apunts Educación Física y Deportes, 87, 99-106.

8. Nikolaidis, P.T. (2014). Age-related differences in countermovement vertical jump in soccer players 8-31 years old: the role of fat-free mass. American Journal of Sports Science and Medicine, 2(2), 60-64.

9. Urzua, R. Von Oetinger, A. Cancino, J. (2009). Potencia aeróbica máxima, fuerza explosiva del miembro inferior y peak de torque isocinético en futbolistas chilenos profesionales y universitarios. Rendimiento en el deporte, 14, 49-52.

10. Sáez de Villarreal, E. Suarez-Arrones, L. Requena, B. Haff, G. Ferrete, C. (2015). Effects of plyometric and sprint training on physical and technical skill performance on adolescent soccer players. Journal of Strength and Conditioning Research, 29(7), 1894-1903.

11. Yanci, J. Los Arcos, A. (2014). Differences in muscle strength and leg asymmetries in elite runners and cyclists. International SportMedicine Journal, 15(3), 285-297.

12. Pääsuke, M. Ereline J. Gapeyeva, H. (2001). Knee extension strength and vertical jumping performance in Nordic combined athletes. The Journal of Sports Medicine and Physical Fitness, 41(3), 354-361.

13. Salaj, S. Markovic, G. (2011). Specificity of jumping, sprinting, and quick change of direction motor abilities. Journal of Strength and Conditioning Research, 25(5), 1249-1255.

14. Chelly, M. Fathloun, M. Cherif, N. Ben Amar, M. Tabka, Z. Van Praagh, E. (2013). Effects of a back squat training program on leg power, jump, and sprint performances in junior soccer players. Journal of Strength and Conditioning Research, 23(8), 2241-2249. 
15. Yanci, J. Los Arcos, A. Cámara, J. (2014). Physical characteristics and unilateral differences of vertical and horizontal jump in elite soccer players. Journal of Sport and Health Research, 6(3), 217-226.

16. Asadi, A. (2013). Effects of in-season short-term plyometric training on jumping and agility performance of basketball players. Sport Sciences for Health, 9(3), 133-137.

17. Bueno, J. (2010). Observational analysis of jumping and landing techniques in elite male soccer players. Tesis Doctoral, Universidad Pablo de Olavide, Sevilla, España.

18. López, I. (2005). Propuesta de trabajo para la mejora de la velocidad en el jugador de baloncesto. Efdeportes, 82, http:// www.efdeportes.com/efd82/balonc.htm.

19. Requena, B. García, I. Requena, F. Bressel, E. Sáez de Villarreal, E. Cronin, J. (2012). Association between traditional standing vertical jumps and a soccer-specific vertical jump. European Journal of Sport Science, 14(S1), 1-8.

20. Hoffman, J.R. Tenenbaum, G. Maresh, C.M. Kraemer, W.J. (1996). Relationship between athletic performance tests and playing time in elite college basketball players. Journal of Strength and Conditioning Research, 10(2), 67-71.

21. Lara, A. Alegre, M.L. Abián, J. Jiménez, L. Ureña, A. Aguado, X. (2006). The selection of a method for estimating power output from jump performance. Journal of Human Movement Studies, 50, 399-410.

22. Huertas, F. Pérez, P. (2006). Evaluación cineantropométrica y condicional del futbolista en diferentes edades. Motricidad: Revista de Ciencias de la Actividad Física y del Deporte, 15, $1-7$.

23. Hellín, M.D. Luis, V. Santiago, J. Navarrete, G. Gómez-Valades, J.M. Murillo, D.B. Sabido, R. (2014). Diferencias en tests isométricos de fuerza y tests de salto entre jugadores de baloncesto profesionales y amateurs. Cultura Ciencia y Deporte, 26, 155-162.

24. Henry, G.J. Dawson, B. Lay, B.S. Young, W.B. (2013). Relationships between reactive agility movement time and unilateral vertical, horizontal and lateral jumps. Journal of Strength and Conditioning Research. In press.

25. Maulder, P. Cronin, J. (2005). Horizontal and vertical jump assessment: Reliability, symmetry, discriminative and predictive ability. Physical Therapy in Sport, 6(2), 74-82.

26. Ghedini, R. Dal Pupo, J. Pereira, L.M., Monteiro, B. Giovana, S. (2014). Effect of squat depth on performance and biomechanical parameters of countermovement vertical jump. Revista Brasileira de Cineantropometria e Desempenho Humano, 16(6), 658-668.

27. Cowley, H. R. Ford, K. R. Myer, G. D. Kernozek, T. W. Hewett, T. E. (2006). Differences in neuromuscular strategies between landing and cutting tasks in female basketball and soccer athletes. Journal of Athletic Training, 41(1), 67-73.

28. Marques, M.C. Gil, H. Ramos, R.J. Costa, A.M. Marinho, D.A. (2011). Relationships between vertical jump strength metrics and 5 meters sprint time. Journal of Human Kinetics, 29(1), 115-122. 
29. Gomes, M.M. Pereira, G. de Freitas, P.B. Barela, J.A. (2009). Características cinemáticas e cinéticas do salto vertical: Comparação entre jogadores de futebol e basquetebol. Revista Brasileira de Cineantropometria e Desempenho Humano, 11(4), 392-399.

30. Rouis, M. Attiogbé, E. Vandewalle, H. Jaafar, H. Noakes, T.D. Driss, T. (2014). Relationship between vertical jump and maximal power output of legs and arms: Effects of ethnicity and sport. Scandinavian Journal of Medicine and Science in Sports, 25(2), 197207.

31. Miyaguchi, K. Demura, S. (2010). Specific factors that influence deciding the takeoff leg during jumping movements. Journal of Strength and Conditioning Research, 24(9), 25162522 .

32. Newton R.U. Gerber A, Nimphius, S. Shim, J.K. Doan, B.K. Robertson, M. Pearson, D.R., Craig, B.W. Häkkinen, K. Kraemer, W.J. (2006) Determination of functional strength imbalance of the lower extremities. Journal of Strength and Conditioning Research, 20(4), 971-977.

33. Cohen J. (1988). Statistical power analysis for the behavioral sciences. Hillsdale, NJ: Lawrence Erlbaum Associates.

34. Miranda da Rocha, C. Ugrinowitsch, C. Barbanti, V.J. (2005). The specificity of sport training and the vertical jump skill. A study with volleyball and basketball players from different categories. Efdeportes, 84, http://www.efdeportes.com/efd84/saltar.htm

35. Tricoli, V.A.A. Barbanti, V.J. Shinzato, G.T. (1994). Potência muscular em jogadores de basquetebol e voleibol: relação entre dinamometria isocinética e salto vertical. Revista Paulista de Educação Física, 8(2), 14-27.

36. Chaouachi, A. Brughelli, M. Levin, G. Boudhina, N.B.B. Cronin, J. Chamari, K. (2009). Anthropometric, physiological and performance characteristics of elite team-handball players. Journal of Sports Sciences, 27(2), 151-157.

37. Mero, A. Komi, P.V. (1994). EMG, force, and power analysis of sprint-specific strength exercises. Journal of Applied Biomechanics, 10(1), 1-13.

38. Yanci, J. Arcos, A.L. Mendiguchia, J. Brughelli, M. (2014). Relationships between sprinting, agility, one- and two-leg vertical and horizontal. Kinesiology, 46, 194-201.

39. Thomas, C. Mather, D. Comfort, P. (2014). Changes in sprint, change of direction, and jump performance during a competitive season in male lacrosse players. Journal of Athletic Enhancement, 3(5), 1-8.

40. Izquierdo, M. Aguado, X. Gonzalez, R. López, J.L. Häkkinen, K. (1999). Maximal and explosive force production capacity and balance performance in men of different ages. European Journal of Applied Physiology and Occupational Physiology, 79(3), 260-267.

41. Castro-Piñero, J. Ortega, F.B. Artero, E.G. Girela-Rejón, M.J. Mora, J. Sjöström, M. Ruiz, J.R. (2010). Assessing muscular strength in youth: usefulness of standing long jump as a general index of muscular fitness. Journal of Strength and Conditioning Research, 24(7), 1810-1817. 
42. Hamilton, R.T. Shultz, S.J. Schmitz, R.J. Perrin, D.H. (2008). Triple-hop distance as a valid predictor of lower limb strength and power. Journal of Athletic Training, 43(2), 144-151.

Número de referencias totales: 42

Número de referencias de La Revista Iberoamericana de Ciencias de la Actividad Física y del Deporte: 0 